Draft Version JANUARY 13, 2022

Preprint typeset using $\mathrm{LAT}_{\mathrm{E}} \mathrm{X}$ style emulateapj v. 12/16/11

\title{
TESTING THE KERR BLACK HOLE HYPOTHESIS WITH THE CONTINUUM-FITTING AND THE IRON LINE METHODS: THE CASE OF GRS 1915+105
}

\author{
Ashutosh Tripathi ${ }^{1}$, Askar B. Abdikamalov ${ }^{1,2,3}$, Dimitry Ayzenberg ${ }^{4}$, Cosimo Bambi $^{1, \dagger}$, Victoria GrinberG $^{5}$, \\ Honghui Liu ${ }^{1}$, AND Menglei Zhou ${ }^{6}$ \\ Draft version January 13, 2022
}

\begin{abstract}
The continuum-fitting and the iron line methods are currently the two leading techniques for probing the strong gravity region around accreting black holes. In the present work, we test the Kerr black hole hypothesis with the stellar-mass black hole in GRS $1915+105$ by analyzing five disk-dominated RXTE spectra and one reflection-dominated Suzaku spectrum. The combination of the constraints from the continuum-fitting and the iron line methods has the potential to provide more stringent tests of the Kerr metric. Our constraint on the Johannsen deformation parameter $\alpha_{13}$ is $-0.15<\alpha_{13}<0.14$ at $3 \sigma$, where the Kerr metric is recovered when $\alpha_{13}=0$.
\end{abstract}

\section{INTRODUCTION}

The Kerr hypothesis states that all isolated, stationary, and axisymmetric astrophysical (uncharged) black holes $(\mathrm{BHs})$ are described by the Kerr metric. This hypothesis is a consequence of the $\mathrm{BH}$ uniqueness theorems (see, e.g., Chruściel et al. 2012). The Kerr metric is completely determined by two physical parameters: the mass of the $\mathrm{BH} M$ and the spin angular momentum of the $\mathrm{BH}$ $|\vec{J}|$. The Kerr hypothesis holds in general relativity (GR) and some modified gravity theories (Psaltis et al. 2008). However, there are theories in which the Kerr metric is not a solution (see, e.g., Alexander \& Yunes 2009; Kleihaus et al. 2011). Additionally, the introduction of new physics, such as exotic matter fields (Herdeiro \& Radu 2014) or macroscopic quantum effects (Giddings 2017), can also lead to violations of the Kerr hypothesis. Tests of the Kerr hypothesis, and in turn GR, are necessary to gain a full understanding of $\mathrm{BHs}$ and gravity (Bambi 2011, 2017).

In this work, we test the Kerr hypothesis using a combined analysis with two BH electromagnetic observation methods: the continuum-fitting method (Zhang et al. 1997; McClintock et al. 2011, 2014) and X-ray reflection spectroscopy (Brenneman \& Reynolds 2006; Reynolds 2014; Bambi et al. 2020). The source of the electromagnetic radiation in the $\mathrm{BH}$ system is an accretion disk that surrounds the $\mathrm{BH}$ and, in the case studied here, is built up from gas that is siphoned off of a stellar companion. The accretion disk is generally assumed to be geometrically thin, optically thick, and cold (on the order of $1 \mathrm{keV}$ for the stellar-mass BHs). The gas in the disk emits as a

\footnotetext{
${ }^{1}$ Center for Field Theory and Particle Physics and Department of Physics, Fudan University, 200438 Shanghai, China. $\dagger$ E-mail: bambi@fudan.edu.cn

${ }^{2}$ Ulugh Beg Astronomical Institute, Tashkent 100052, Uzbekistan

3 Tashkent Institute of Irrigation and Agricultural Mechanization Engineers, Tashkent 100000, Uzbekistan

${ }^{4}$ Theoretical Astrophysics, Eberhard-Karls Universität Tübingen, D-72076 Tübingen, Germany

${ }^{5}$ European Space Agency (ESA), European Space Research and Technology Centre (ESTEC), Keplerlaan 1, 2201 AZ Noordwijk, The Netherlands

${ }^{6}$ Institut für Astronomie und Astrophysik, Eberhard-Karls Universität Tübingen, D-72076 Tübingen, Germany
}

blackbody, but has a temperature that is dependent on its distance from the $\mathrm{BH}$, and so the overall disk emits as a multi-temperature blackbody. This multi-temperature blackbody radiation is known as the $\mathrm{BH}$ continuum or thermal spectrum. These BH-disk systems also include a corona, which is a region of hotter $(\sim 100 \mathrm{keV})$ plasma that is close to the $\mathrm{BH}$ and the inner part of the accretion disk. Some of the thermal radiation from the disk inverse-Compton scatters off free electrons in the corona, producing a power-law spectrum with a high-energy cutoff. Some of this power-law emission illuminates the disk and is reflected, modifying the original power-law spectrum, most importantly with fluorescent emission lines in the soft X-ray band and a Compton hump that peaks at 20-30 keV (Ross \& Fabian 2005; García \& Kallman 2010). The brightest emission line is the iron $\mathrm{K} \alpha$ complex at $\sim 6 \mathrm{keV}$ and is known as the iron line. The overall reflected emission is known as the reflection spectrum and is studied through X-ray reflection spectroscopy.

The continuum spectrum and the reflection spectrum are both strongly dependent on the properties of the $\mathrm{BH}$ spacetime. Thus, these observations can in principle be used to test the Kerr hypothesis (Torres 2002; Lu \& Torres 2003; Schee \& Stuchlík 2009; Bambi \& Barausse 2011; Johannsen \& Psaltis 2013; Bambi 2013). For this purpose, some of us have developed two XSPEC models (Arnaud 1996) to analyze observed spectra while allowing for a non-Kerr spacetime. The continuum spectrum is modeled through nkbb (Zhou et al. 2019) and the reflection spectrum through relxill_nk (Bambi et al. 2017; Abdikamalov et al. 2019), which is an extension of the relxill package (Dauser et al. 2013; García et al. 2014). With these models, we can employ any well-behaved stationary and axisymmetric BH solution. However, here, and in past work, we primarily focus on solutions that parametrically deform the Kerr spacetime and do not represent any specific non-Kerr solution in GR or a modified gravity theory. By doing so we are performing a null test, i.e. determining if observations are consistent with a null result, which in our case is the Kerr solution and a test of the Kerr hypothesis (see, e.g., Tripathi et al. 2019, 2021a,b). In this work, we use the Johannsen spacetime (Johannsen 2013), which parametrically deforms the Kerr spacetime while still retaining three con- 
stants of the motion as in the Kerr solution. For simplicity, we here only study the strongest of the deformation parameters present in the Johannsen spacetime; however, there are an infinite number of deformation parameters present.

In this paper, we apply the continuum-fitting and iron line methods to the BH in GRS $1915+105$. This source was discovered by Granat satellite in 1992 (CastroTirado et al. 1992). It is identified as a microquasar, as it displays radio jets (Mirabel \& Rodríguez 1999). GRS $1915+105$ shows remarkable variability across the whole electromagnetic spectrum (Belloni et al. 2000; Klein-Wolt et al. 2002). Zhang et al. (1997) reported a very high spin of this source and claimed it to be the reason for its exceptional variability and presence of relativistic jets. Observation from BeppoSAX clearly identified the broad Iron $\mathrm{K} \alpha$ line around $6.4 \mathrm{keV}$ (Martocchia et al. 2002). The most recent measurements of the mass and distance of this source are $12.4_{-1.8}^{+2.0} M_{\odot}$ and $8.6_{-1.6}^{+2.0} \mathrm{kpc}$, respectively (Reid et al. 2014). The broad line emission has also been observed with other X-ray missions and high spin measurements are reported using the continuum fitting method for RXTE data (McClintock et al. 2006) and X-ray reflection spectroscopy for Suzaku (Blum et al. 2009) and NuSTAR observations (Miller et al. 2013). Zhang et al. (2019a,b) analyzed the NuSTAR and Suzaku observations, respectively, to test GR by employing the Johannsen metric.

This paper is organized as follows. In Section 2, we briefly describe the relxill_nk and nkbb models. In Section 3, we describe the RXTE and Suzaku observations, our data reduction, and the spectral analysis with relxill_nk and nkbb. We discuss our results and conclude in Section 4. The Johannsen metric employed in our spectral analysis is reported in the appendix.

\section{THE RELXILL_NK AND NKBB MODELS}

Here we briefly review the relxill_nk and nkbb models. Since ray-tracing calculations, which give us the spectrum of a disk away from the source, require too much time and resources for the real-time data analysis stage, both models use the transfer function formalism proposed by Cunningham (Cunningham 1975), which allows us to tabulate all the necessary details of the spacetime metric to a FITS (Flexible Image Transfer System) file. The tabulated data can then be extracted for use in the data analysis phase.

With the transfer function formalism, the observed flux can be written as

$$
\begin{aligned}
F_{o}\left(E_{o}\right)=\frac{1}{D^{2}} \int_{R_{\mathrm{in}}}^{R_{\mathrm{out}}} \int_{0}^{1} & \frac{\pi r_{e} g^{2} f\left(g^{*}, r_{e}, \iota\right)}{\sqrt{g^{*}\left(1-g^{*}\right)}} \\
& \times I_{e}\left(E_{e}, r_{e}, \theta_{e}\right) d g^{*} d r_{e},
\end{aligned}
$$

where $D$ denotes the distance from the source to the observer's screen, $R_{\text {in }}\left(R_{\text {out }}\right)$ is the inner (outer) boundary of the accretion disk, $r_{e}$ is the radial coordinate of the emitting rings in the accretion disk, $g=E_{o} / E_{e}$ is the redshift factor, defined as the ratio of the energy of photons registered on the observer's screen $\left(E_{o}\right)$ and at the emission point in the rest frame of the disk's gas $\left(E_{e}\right)$. $I_{e}\left(E_{e}, r_{e}, \theta_{e}\right)$ is the specific intensity at the emission point on the accretion disk at the energy $E_{e}$, the radial coordinate $r_{e}$, and the radiation angle $\theta_{e}$ (angle between the emitted photons and the normal to the disk). The transfer function $f$ has the following form

$$
f\left(g^{*}, r_{e}, \iota\right)=\frac{1}{\pi r_{e}} g \sqrt{g^{*}\left(1-g^{*}\right)}\left|\frac{\partial(X, Y)}{\partial\left(g^{*}, r_{e}\right)}\right| .
$$

Here $\left|\partial(X, Y) / \partial\left(g^{*}, r_{e}\right)\right|$ represents the Jacobian, $X$ and $Y$ are the Cartesian coordinates in the observer's screen, and $g^{*}$ is the relative redshift factor, which is written as

$$
g^{*}=\frac{g-g_{\min }}{g_{\max }-g_{\min }} \in[0,1] .
$$

where $g_{\min }=g_{\min }\left(r_{e}, \iota\right) \quad\left(g_{\max }=g_{\max }\left(r_{e}, \iota\right)\right)$ denotes the minimum (maximum) value of the redshift factor $g$ for a given radial coordinate $r_{e}$ and the viewing angle $\iota$ of an observer. Thus, the transfer function formalism can separate the calculations related to the spectrum appearing on the surface of the disk in the rest-frame of the emitting gas from those that transmit the emitted spectrum from the emission point to a distant observer, taking into account the effects of the spacetime metric. This separation results in a fast calculation of the observed spectrum without the need to recalculate all the photon trajectories.

In both models, the transfer functions are precalculated for a three-dimensional grid, which consists of the BH spin parameter $a_{*}$, the Johannsen spacetime with deformation parameter $\alpha_{13}$ (Johannsen 2013), and the viewing angle $\iota$, and are tabulated in the FITS table. The parameter ranges of the grid are selected so that the entire parameter space is effectively covered. At the data analysis stage, the transfer function values corresponding to the set of model input parameters are passed to Eq. 1 using interpolation methods.

relxill_nk adopts the model suite xillver (García \& Kallman 2010; García et al. 2014) for the calculation of the reflection spectrum of the accretion disk at the emission point, namely for calculating $I_{e}\left(E_{e}, r_{e}, \theta_{e}\right)$. Therefore, all model parameters of xillver are included in relxill_nk.

nkbb assumes that the accretion disk is in local thermal equilibrium, and the emission at each point of the disk is like a blackbody. The local specific intensity of the disk radiation is taken as (in units in which $h=c=k_{\mathrm{B}}=1$ )

$$
I_{e}\left(E_{e}\right)=\frac{2 E_{e}^{3}}{f_{\mathrm{col}}^{4}} \frac{\Upsilon}{\exp \left(\frac{E_{e}}{T_{\mathrm{col}}}\right)-1},
$$

where $f_{\text {col }}$ is the color factor (or hardening factor), which is introduced to account for non-thermal effects occurring mainly due to electron scattering in the disk atmosphere. $\Upsilon$ is a function of the angle between the emitted photons from the disk surface and the normal to the disk. Isotropic emission $(\Upsilon=1)$ and limb-darkened emission $\left(\Upsilon=\frac{1}{2}+\frac{3}{4} \cos \theta_{e}\right)$ are the two most common options. $T_{\text {col }}(r)=f_{\text {col }} T_{\text {eff }}$ is the color temperature, and $T_{\text {eff }}(r)$ is the effective temperature at every radius defined from the relation $\mathcal{F}(r)=\sigma T_{\text {eff }}^{4}$, where $\sigma$ denotes the StefanBoltzmann constant and $\mathcal{F}(r)$ is the time-averaged energy flux emitted by the surface of the Novikov-Thorne disk defined as (see, e.g., Bambi 2012)

$$
\mathcal{F}(r)=\frac{\dot{M}}{4 \pi M^{2}} F(r) \text {. }
$$


Here $M$ and $\dot{M}$ are, respectively, the $\mathrm{BH}$ mass and the mass accretion rate, and $F(r)$ is a dimensionless function that depends on the spacetime metric. In the end, the BH mass $M$, the mass accretion rate $\dot{M}$, the $\mathrm{BH}$ distance $D$, the $\mathrm{BH}$ spin $a_{*}$, the Johannsen spacetime deformation parameter $\alpha_{13}$, the observer's viewing angle $\iota$, the function $\Upsilon$, and the color factor $f_{\text {col }}$ are the parameters of the nkbb model.

Fig. 1 shows how the parameters $M, \dot{M}, D, \iota, a_{*}$, and $\alpha_{13}$ affect the thermal spectrum. It is clear that the $\mathrm{BH}$ spin parameter $a_{*}$ and the Johannsen deformation parameter $\alpha_{13}$ have a very similar impact on the shape of the spectrum. The high-energy cutoff of the spectrum is indeed determined by the inner edge of the disk, which is assumed to be at the innermost stable circular orbit (ISCO). When the BH mass is known, the ISCO is determined by $a_{*}$ and $\alpha_{13}$. Apart from determining the ISCO radius, the impact of $a_{*}$ and $\alpha_{13}$ on the thermal spectrum of the disk is very weak. As already pointed out in Kong et al. (2014), in such a situation there is a strong correlation between the estimates of the spin and the deformation parameters and - as we will verify in the next section - our constraints on the plane $a_{*}$ vs. $\alpha_{13}$ will appear with the typical banana-shape of two degenerate parameters.

\section{DATA REDUCTION AND ANALYSIS}

While GRS 1915+105 has been observed many times and by different X-ray missions, there are not many observations of this source suitable for the continuumfitting method and the analysis of the reflection features of the disk. In part, this is because GRS $1915+105$ is quite a peculiar and complicated source. Its mass accretion rate is often too high for maintaining a thin accretion disk, so our models based on Novikov-Thorne disks are not applicable. To the best of our knowledge, there are only the RXTE data studied in McClintock et al. (2006) suitable for the continuum-fitting method and the Suzaku observation analyzed in Blum et al. (2009) suitable for X-ray reflection spectroscopy. These will be the data analyzed in our study.

\subsection{RXTE data}

For data selection, we follow McClintock et al. (2006) which used data taken with the Proportional Counter Array (PCA) onboard the Rossi X-ray Timing Explorer (RXTE) mission in which the source is in a thermal dominant state. A thermal dominant state (Remillard \& McClintock 2006) is defined as a period with a majority $(>75 \%)$ of the emission from the accretion disk over the 2.0-20.0 keV energy band, low root mean square (RMS) variability $(<0.075$ integrated over $0.1-1.0 \mathrm{~Hz})$ and absence or very weak presence of quasi-periodic oscillations (QPOs) (amplitude less than 0.005\%). By screening all RXTE archival data of this source with thermal dominance criteria, McClintock et al. (2006) identified 20 observations for further analysis. Out of these 20 observations, we select five key low-luminosity observations. This is because, for the continuum-fitting method used in this work, the luminosity should be less than $30 \%$ of the Eddington luminosity. Tab. 1 shows the basic details of the five RXTE observations used in this work.

\subsubsection{Data reduction}

For data reduction, we follow Zhou et al. (2020) which uses a standard data reduction approach. We only include data from PCU-2 because it is the best calibrated PCU and was always operating. We extract the PCA spectra in the standard2f mode. We also ignore the data taken within 10 minutes of the South Atlantic Anomaly (SAA). Using pcacorr tool (García et al. 2014), the calibration of the PCA data is further improved. As the count rates are high, the systematic uncertainty of $0.1 \%$ is added to all PCA data following García et al. (2014). We rebin the data such that the signal-to-noise $(\mathrm{S} / \mathrm{N})$ ratio is 10 . We use data in the energy range $3.0-45.0 \mathrm{keV}$ for all five observations for further spectral analysis.

\subsubsection{Data analysis}

The spectral analysis work in this paper is done using XSPEC v12.11.1 (Arnaud 1996). We consider the abundances from Wilms et al. (2000) and the cross-sections from Verner et al. (1996). For galactic absorption, tbabs (Wilms et al. 2000) is used with column density frozen at $8 \times 10^{22} \mathrm{~cm}^{-2}$ following Abdikamalov et al. (2020). We note that the exact value of the column density has a very weak impact on the final fit. We start our analysis by fitting the data with an absorbed power-law continuum (powerlaw) and a multi-temperature disk blackbody (diskbb) (Mitsuda et al. 1984). There are several narrow absorption features present in the energy range of $6.4-8.4 \mathrm{keV}$ which is also consistent with Kotani et al. (2000). Recently, such narrow absorption lines have also been observed with NICER: Neilsen et al. (2020) reported the detection of multiple absorption features in the energy range 6.4-8.4 keV. Such absorption features can be interpreted as different iron absorption lines. In our case, given the limited resolution of the PCA spectra, we follow McClintock et al. (2006) and we add a broad Gaussian absorption line (gabs) with a limited width of $0.5 \mathrm{keV}$ and energy restricted between 6.3 and $7.5 \mathrm{keV}$.

We also add smedge (Ebisawa et al. 1994) to model the broad $\mathrm{Fe}$ absorption edge with the energies to vary between 6.9 and $9.0 \mathrm{keV}$. The addition of these two components significantly improves the fit.

After identifying the line components, we replace the Newtonian disk model diskbb with nkbb (Zhou et al. 2019), which calculates relativistic thermal spectra of accretion disks in the Johannsen metric. The spacetime parameters are the $\mathrm{BH}$ mass, the (dimensionless) $\mathrm{BH}$ spin $a_{*}$, and the deformation parameter $\alpha_{13}$. The other model parameters are the distance $(D)$, the inclination angle of the disk with respect to the line of sight of an observer $(\iota)$, and the mass accretion rate $(\dot{M})$. Due to degeneracies, it is not possible to measure these parameters simultaneously by fitting the data of a source. So, we need independent measurements of $M, \iota$, and $D$. The fit will provide the measurements of $a_{*}, \dot{M}$, and $\alpha_{13}$. We freeze the mass of the source at $M=12.4 M_{\odot}$ and distance at $D=8.6 \mathrm{kpc}$ (Reid et al. 2014). Here, we freeze the value of inclination angle $\iota$ to $65^{\circ}$ measured by high resolution radio images (Fender et al. 1999) assuming that the jet of the source is parallel to the $\mathrm{BH}$ spin and orthogonal to the accretion disk. We assume limb darkening emission (lflag=1). We freeze the spectral hardening factor $f_{\text {col }}$ to 1.7, which is the most widely used value for a stellarmass $\mathrm{BH}$ with an Eddington-scaled accretion luminosity 

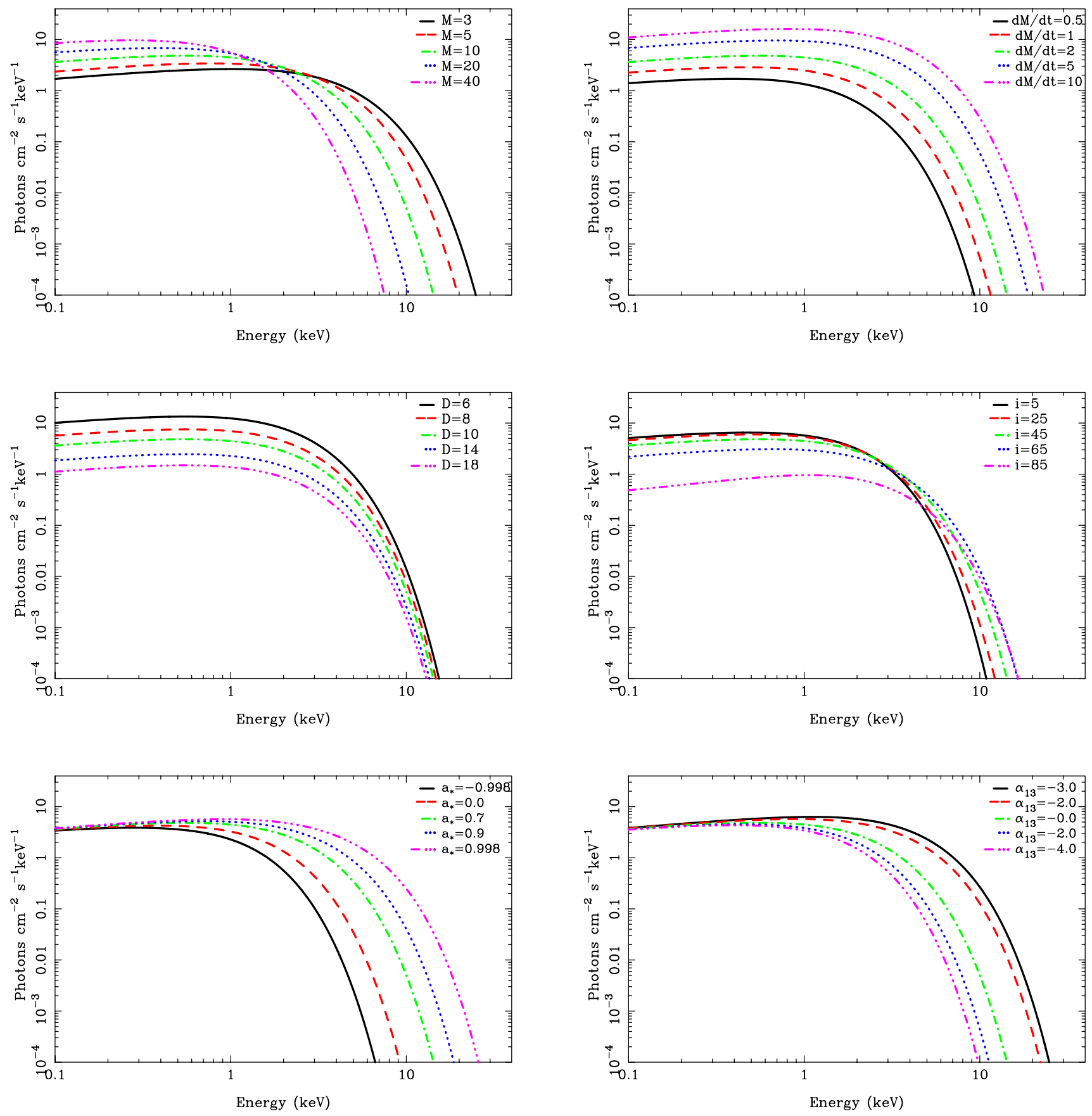

FIG. 1. - Impact of the $\mathrm{BH}$ mass $M$, the mass accretion rate $\dot{M}$, the distance $D$, the inclination angle of the disk $\iota$, the BH spin parameter $a_{*}$, and the deformation parameter $\alpha_{13}$ on the thermal spectra of accretion disks as calculated by nkbb. $M$ is in units of $M_{\odot} ; \dot{M}$ is in units of $10^{18} \mathrm{~g} / \mathrm{s} ; D$ is in units of $\mathrm{kpc}$; $\iota$ in deg. For all spectra, we assume $f_{\text {col }}=1.7$ and $\Upsilon=1$. The default values of the model parameters are: $M=10 M_{\odot}, \dot{M}=2 \cdot 10^{18} \mathrm{~g} / \mathrm{s}, D=10 \mathrm{kpc}, \iota=45^{\circ}, a_{*}=0.7$, and $\alpha_{13}=0$.

around $10 \%$ (Shimura \& Takahara 1995). As we freeze $M, \iota$, and $D$, the normalization of nkbb is frozen to 1 .

We found that, in some cases, the power-law continuum contributes significantly to the flux at energies below $5 \mathrm{keV}$. This should not be the case as the power-law emission is produced by Comptonization of soft disk photons in the corona. So, we consider more realistic models. We try nthcomp, comptt, and simplcutx. We find that comptt (Titarchuk 1994) always fits the data better than the other two models, so we use it to describe the coronal spectrum for further analysis.

As pointed out in McClintock et al. (2006), these low luminosity observations give high $\chi^{2}$ values $(\sim 1.5)$. In order to improve the fit, we follow McClintock et al. (2006) and add a sharp edge feature (edge in XSPEC) with energy allowed to vary between 8 and $13 \mathrm{keV}$ and we refit the spectra. Note that edge improves $\chi^{2}$ statistics, but it does not have any significant impact on the measurement of $a_{*}, \dot{M}$, and $\alpha_{13}$, in agreement with McClintock et al. 


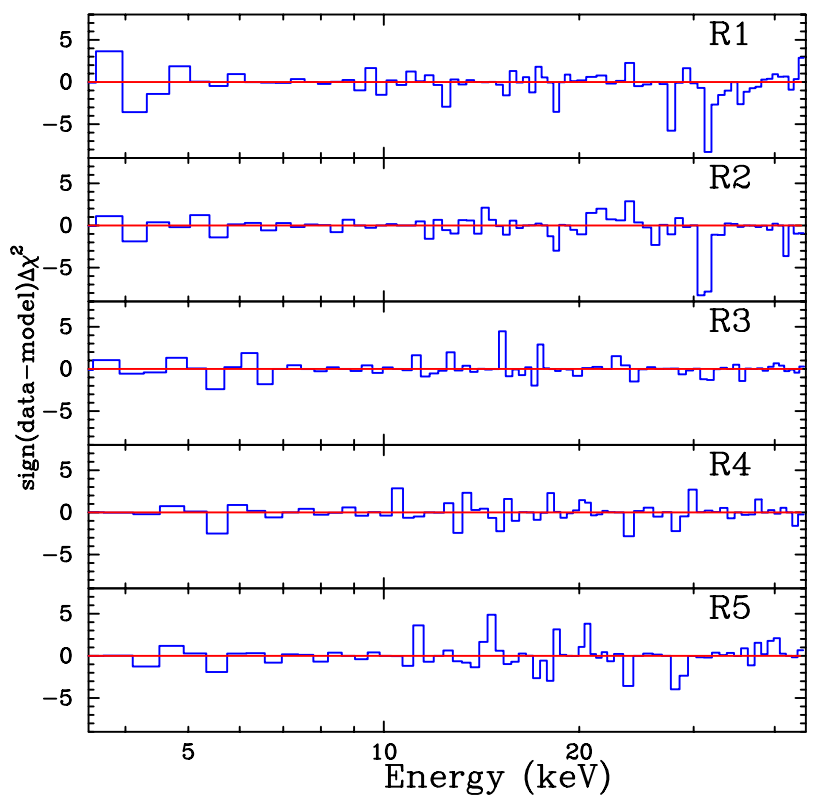

FIG. 2.- Residuals of the individual fits of the five RXTE observations of GRS 1915+105.

(2006). In XSPEC jargon, the model eventually reads as

$$
\text { tbabs } \times \text { smedge } \times \text { gabs } \times \text { edge } \times(\text { comptt }+n k b b) .
$$

The results of our fits are summarized in Tab. 2, which shows both the measurements of the fits of the individual observations (R1, R2, R3, R4, and R5) and of the fit of all observations together (R1-R5, last column). Fig. 2 shows the residuals of the individual fits.

\subsection{Suzaku data}

GRS 1915+105 was observed by Suzaku for around $117 \mathrm{ks}$ (Obs. ID 402071010) on 7 May 2007. Tab. 1 shows the details of that observation. The source was in low/hard flux state. Blum et al. (2009) first reported the spectral analysis of this data and measured the $\mathrm{BH}$ spin $a_{*}>0.98$ (assuming the Kerr metric).

\subsubsection{Data reduction}

Here we follow Tripathi et al. (2020a) for data reduction. Out of the four XIS instruments, we use data only from XIS1 as two of the units were turned off during observation and the data from the fourth XIS unit is not suitable for spectral studies as it was running in timing mode during the observation. The unprocessed data of the aforementioned observation ID is downloaded from the HEASARC website and processed to filtered files using aepipeline module, distributed as part of the HEASOFT package, and calibration database CALDB version 20151005. In order to minimize the effect of pile-up, the source region is taken as an annulus with an inner radius of 78 arcsec and an outer radius of 208 arcsec. As background region, we select an annulus with the source at the center and the inner and outer radii of 208 and 278 arcsec, respectively. The redistribution matrix file is created using xisrmfgen and the corresponding ancillary file is produced using xissimarfgen. A net exposure of $29 \mathrm{ks}$ is obtained after screening. Finally, the source spectrum is rebinned to a minimum of 25 counts per bin in order to apply $\chi^{2}$ statistics. We only consider data in the energy range $2.3-10.0 \mathrm{keV}$ for spectral analysis in order to avoid calibration issues.

Data from the PIN detector are used for the HXD instrument. The raw data is processed using aepipeline. The spectrum, ancillary file, and response matrix file are obtained using the ftool hxdpinxbpi and CALDB. The net exposure of $53 \mathrm{ks}$ is obtained and the data is rebinned to 25 counts per bin. For spectral analysis, data in the energy range 12.0-55.0 keV are used. During the spectral fits, the cross-calibration constant between XIS1 and PIN data is kept free because of XIS1 being affected by pile-up.

GRS $1915+105$ is believed to be a highly variable source but its flux and hardness did not vary much during this particular observation (see Tripathi et al. 2020a). The time-averaged spectrum is thus used without resolving the data in the time or flux domain.

\subsubsection{Data analysis}

The spectrum of this particular observation is simple and can be fit with coronal and reflection components, modified by galactic absorption (Blum et al. 2009; Abdikamalov et al. 2020). In XSPEC, the model reads as

\section{tbabs $\times$ (comptt+relxillCp_nk)}

tbabs describes the Galactic absorption (Wilms et al. 2000) and the column density $N_{\mathrm{H}}$ is left free in the fit. comptt describes the Comptonization of seed photons in the hot plasma of the corona (Titarchuk 1994). The model includes three parameters which defines the Comptonized spectrum: the temperature of the seed photons $T_{0}$, the temperature of the plasma $T_{\mathrm{e}}$, and the plasma optical depth $\tau$. relxillCp_nk describes the reflection spectrum of the accretion disk in the Johannsen metric with possible non-vanishing deformation parameter $\alpha_{13}$ (Bambi et al. 2017; Abdikamalov et al. 2019). We model the emissivity profile with a broken power-law, so we have three free parameters: the inner emissivity index $q_{\text {in }}$, the outer emissivity index $q_{\text {out }}$, and the breaking radius $R_{\text {br }}$. Since we use comptt to describe the continuum from the corona, the reflection fraction in relxillCp_nk is set to -1 and the model returns only the reflection spectrum. The electron temperature in relxillCp_nk is linked to $T_{\mathrm{e}}$ in comptt.

The results of the fit are summarized in Tab. 3. Fig. 3 shows the best-fit model and the data to the best-fit model ratio.

\section{DISCUSSION AND CONCLUSIONS}

In the present paper, we have analyzed five diskdominated RXTE data and one reflection-dominated Suzaku spectrum of the BH in GRS 1915+105 employing the models nkbb and relxill_nk. Our goal is to test the Kerr hypothesis by combining the study of the thermal component and the reflection features. We note that similar studies were reported in Tripathi et al. (2021a) and in Zhang et al. (2021). Tripathi et al. (2021a) analyzed a NuSTAR observation of GX 339-4 in which spectrum simultaneously shows a strong thermal component and prominent reflection features. However, the $\mathrm{BH}$ mass and the distance of the source are not known for GX 339-4, so they were inferred in the fit while the continuum-fitting method requires to know the values of these two parameters in order to estimate the $\mathrm{BH}$ spin (and the possible 
Tripathi et al.

\begin{tabular}{lccccc}
\hline \hline Obs. & Mission & Observation ID & Observation Date & Exposure (ks) & Counts/s \\
\hline \hline R1 & RXTE & $10408-01-20-00$ & 1996 Jul 3 & 3.3 & 1349 \\
R2 & RXTE & 10408-01-20-01 & 1996 Jul 3 & 2.9 & 1381 \\
R3 & RXTE & $30703-01-13-00$ & 1998 Mar 29 & 4.7 & 1230 \\
R4 & RXTE & $70702-01-36-00$ & 2003 Jan 1 & 2.3 & 1518 \\
R5 & RXTE & $80701-01-34-00$ & 2003 Nov 24 & 3.2 & 1496 \\
\hline S1 & Suzaku & 402071010 & 2007 May 27 & 28.9 & 84 \\
\hline \hline
\end{tabular}

TABLE 1

Summary of the six observations of GRS 1915+105 analyzed in the present study.

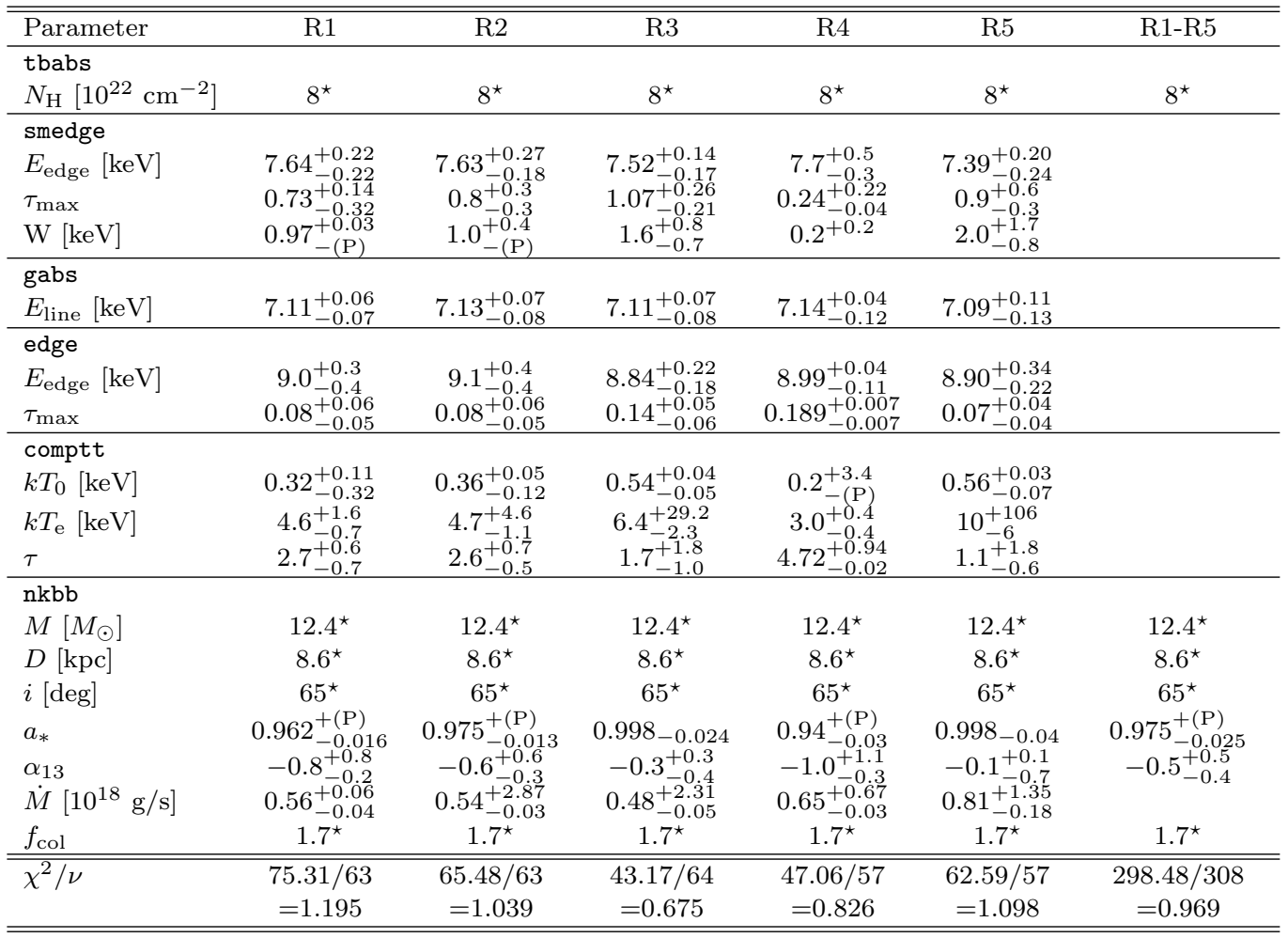

TABLE 2

Best-fit values from the analysis of the RXTE observations of GRS $1915+105$ with the model tbabs $\times$ smedge $\times$ gabs $\times$ edge $\times$ (comptt+nkbb). We show the results of the fits of the individual observations (R1, R2, R3, R4, and R5) and of the fit of all observations together (R1-R5, last column). The reported uncertainties correspond to $90 \%$ confidence level for one relevant parameter $\left(\Delta \chi^{2}=2.71\right)$. ${ }^{\star}$ indicates that the parameter is frozen in the fit. (P) means that the $90 \%$ confidence level limit is not in the parameter range allowed by the model (the maximum value of $a_{*}$ is 0.998 , the minimum value of $W$ is $0.2 \mathrm{keV}$, and the minimum value of $k T_{0}$ is $0.01 \mathrm{keV}$ ).

deformation parameter). Zhang et al. (2021) analyzed three NuSTAR observations of the BH in GRS 1716-249 in the hard-intermediate state where, again, we simultaneously see a strong thermal spectrum and prominent reflection features. For GRS 1716-249, the distance is known, while for the BH mass we only have a lower limit and its value was thus inferred by the fit. The case of GRS 1915+105 studied in the present work is thus somewhat different. The BH mass and distance are both known (Reid et al. 2014). Moreover, we have five RXTE observations in which we see a very strong thermal component and we do not see any reflection feature, and one Suzaku spectrum with strong reflection features and a negligible thermal component.

Let us start with the five RXTE observations analyzed with nkbb. First, we analyzed every observation separately (R1, $\mathrm{R} 2, \mathrm{R} 3, \mathrm{R} 4$, and $\mathrm{R} 5$ ) and then we fit the data of all observation together (fit R1-R5). The best- fit values of these six fits are reported in Tab. 2. We note that the line energy in edge, smedge, and gabs are consistent among the five individual fits. We always recover a high value of the $\mathrm{BH}$ spin parameter, while the estimate of the deformation parameter includes the Kerr metric $\alpha_{13}=0$ but allows for negative values of $\alpha_{13}$. Fig. 4 shows the constraints on $a_{*}$ and $\alpha_{13}$ of the six fits. The combined analysis of the five observations provide the following constraint on $\alpha_{13}$ (90\% confidence level)

$$
\alpha_{13}=-0.5_{-0.4}^{+0.5} .
$$

This constraint is somewhat weaker than the typical constraints obtained from the analysis of the reflection features with relxill_nk; see, e.g., Tab. 5 in Zhang et al. (2021). However, it is stronger than the constraint on $\alpha_{13}$ inferred by the study of the thermal spectrum of LMC X-1 with nkbb in Tripathi et al. (2020b). While in both studies (here and for LMC X-1) we clearly see 


\begin{tabular}{lc}
\hline \hline Parameter & Value \\
\hline tbabs & \\
$N_{\mathrm{H}}\left[10^{22} \mathrm{~cm}^{-2}\right]$ & $8.35_{-0.18}^{+0.17}$ \\
\hline comptt & \\
$k T_{0}[\mathrm{keV}]$ & $0.23_{-0.05}^{+0.08}$ \\
$k T_{\mathrm{e}}[\mathrm{keV}]$ & $33_{-8}^{+8}$ \\
$\tau$ & $0.71_{-0.09}^{+0.22}$ \\
\hline relxillCp_nk & \\
$q_{\text {in }}$ & $8.9_{-1.4}^{+(\mathrm{P})}$ \\
$q_{\mathrm{out}}$ & $0.00^{+0.21}$ \\
$R_{\mathrm{br}}\left[r_{\mathrm{g}}\right]$ & $7.8_{-1.7}^{+3.4}$ \\
$a_{*}$ & $0.998_{-0.012}^{+0.0}$ \\
$i$ deg] & $73.3_{-1.6}^{+3.06}$ \\
$\Gamma$ & $2.57_{-0.05}^{+0.06}$ \\
$\log \xi[\mathrm{erg} \mathrm{cm} \mathrm{s}$ & $2.77_{-0.06}^{+0.09}$ \\
$A_{\mathrm{Fe}}$ & $0.82_{-0.12}^{+0.13}$ \\
$\alpha_{13}$ & $0.00_{-0.10}^{+0.14}$ \\
\hline$C_{\mathrm{HXD}}$ & $1.922_{-0.024}^{+0.018}$ \\
\hline$\chi^{2} / \nu$ & $2281.51 / 2206$ \\
& $=1.03423$ \\
\hline
\end{tabular}

TABLE 3

Best-fit values from the analysis of the Suzaku observation of GRS $1915+105$ with the model tbabs $\times$ (comptt+relxillCp_nk). The reported uncertainties correspond to $90 \%$ confidence level for one relevant parameter $\left(\Delta \chi^{2}=2.71\right)$.

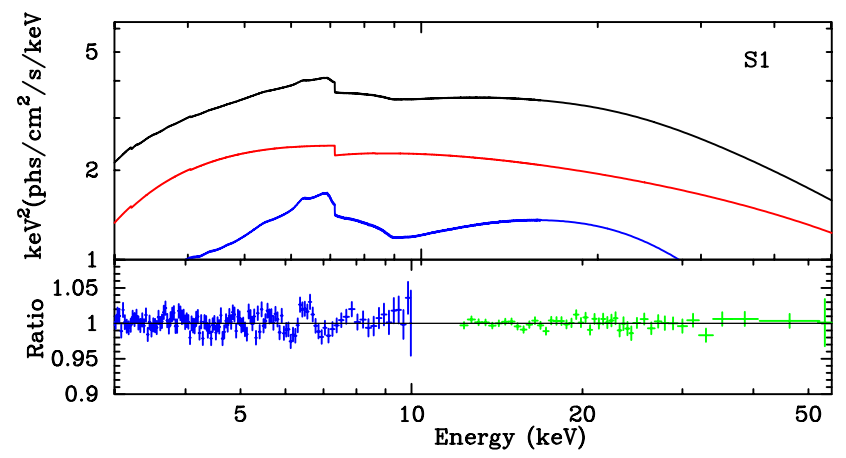

FIG. 3.- Best-fit model (upper quadrant) and data to the best-fit model ratio (lower quadrant) in the analysis of the Suzaku observation of GRS 1915+105. In the upper quadrant, we show the total spectrum (black curve), the relxillCp_nk component (red curve), and the comptt component (blue curve). In the lower quadrant, blue crosses indicate the XIS data and green crosses are for the PIN data.

a strong correlation between the estimate of $a_{*}$ and $\alpha_{13}$, here we recover a higher value of the $\mathrm{BH}$ spin, which helps to constrain the deformation parameter $\alpha_{13}$ because the parameter space is smaller (the gray region in Fig. 4 is ignored in our analysis because the spacetimes have pathological properties there; see the appendix for more details).

We note that the fits in Tab. 2 and the constraints in Fig. 4 are obtained by freezing the values of the $\mathrm{BH}$ mass $M$, viewing angle $\iota$, and source distance $D$ as shown in Tab. 2. However, these three quantities are known with a finite uncertainty. In we plug a lower/higher value for the BH mass $M$ (without changing the values of $\iota$ and $D$ ), the valley of the minimum of $\chi^{2}$ moves to the left/right in the $a_{*}$ vs. $\alpha_{13}$ plane. Similarly, if we employ a lower/higher value of the inclination angle $\iota$ (without changing the values of $M$ and $D$ ), the valley of the minimum of $\chi^{2}$ moves to the right/left in the $a_{*}$ vs. $\alpha_{13}$ plane. Last, if we use a lower/higher value of the source distance $D$ (without changing the values of $M$ and $\iota$ ), the valley of the minimum of $\chi^{2}$ moves to the right/left in the $a_{*}$ vs. $\alpha_{13}$ plane. In order to take the uncertainties of $M, \iota$, and $D$ into account, we can leave these three parameters free in $n k b b$ and we calculate the $\chi^{2}$ as

$$
\chi_{\text {tot }}^{2}=\chi_{\mathrm{RXTE}}^{2}+\frac{\left(M_{*}-M\right)^{2}}{\sigma_{M}^{2}}+\frac{\left(\iota_{*}-\iota\right)^{2}}{\sigma_{\iota}^{2}}+\frac{\left(D_{*}-D\right)^{2}}{\sigma_{D}^{2}},
$$

where $\chi_{\mathrm{RXTE}}^{2}$ is the $\chi^{2}$ contribution from the RXTE data; $M, \iota$, and $D$ are free parameters in the model; $M_{*}=12.4 M_{\odot}, \sigma_{M}=2 M_{\odot}, \iota=65^{\circ}, \sigma_{\iota}=10^{\circ}$, $D_{*}=8.6 \mathrm{kpc}$, and $\sigma_{D}=2.0 \mathrm{kpc}$, following the measurements of $M, D$, and $\iota$ in Reid et al. (2014) and Fender et al. (1999). The last three terms on the right hand side of Eq. (7) are intended to incorporate, in a very simple way, the priors on those parameters. With this procedure, we find the constraints on $a_{*}$ and $\alpha_{13}$ reported in Fig. 5 .

The best-fit values from the Suzaku fit are reported in Tab. 3. This observation was already analyzed with an older version of relxill_nk in Zhang et al. (2019b) and therefore we do not further discuss this fit here. We note that the $90 \%$ confidence level measurement of the Johannsen deformation parameter $\alpha_{13}$ is

$$
\alpha_{13}=0.00_{-0.10}^{+0.14} \text {. }
$$

This is quite a stringent constraint, especially if compared with the constraints from the five disk-dominated spectra inferred with nkbb. While every constraint depends on the quality of the data and the spectrum of the source during a particular observation, it is surely true that the analysis of the reflection features is a more powerful method to probe the strong gravity region around a BH than the continuum-fitting method. The thermal spectrum has indeed a very simple shape, it is just a multi-temperature blackbody spectrum, which can be fit well by a number of different combinations of the model parameters. The constraint on the BH spin and the deformation parameter from the analysis of this Suzaku spectrum are shown in Fig. 6.

Last, we want to combine the analysis of the $R X T E$ and Suzaku data together ${ }^{7}$, taking the uncertainties on $M$ and $D$ into account (the value of the viewing angle $\iota$ can be determined by the fit of the reflection features in the Suzaku spectrum and the parameter $\iota$ in nkbb will be linked to that in relxill_nk). We proceed as before

7 Since the RXTE and Suzaku observations are not simultaneous, we do not have cross-calibration constants between the RXTE and Suzaku instruments. However, this does not introduce any calibration uncertainty. The normalization of the flux is not important in the analysis of the Suzaku data because all details are in the shape of the spectrum (and, indeed, we can analyze the data without any knowledge of the $\mathrm{BH}$ mass and distance). For the analysis of the thermal spectrum in the RXTE data, the normalization of the flux is important, but here the uncertainty is dominated by the uncertainties on the $\mathrm{BH}$ mass and distance, while the calibration uncertainty is subdominant. 

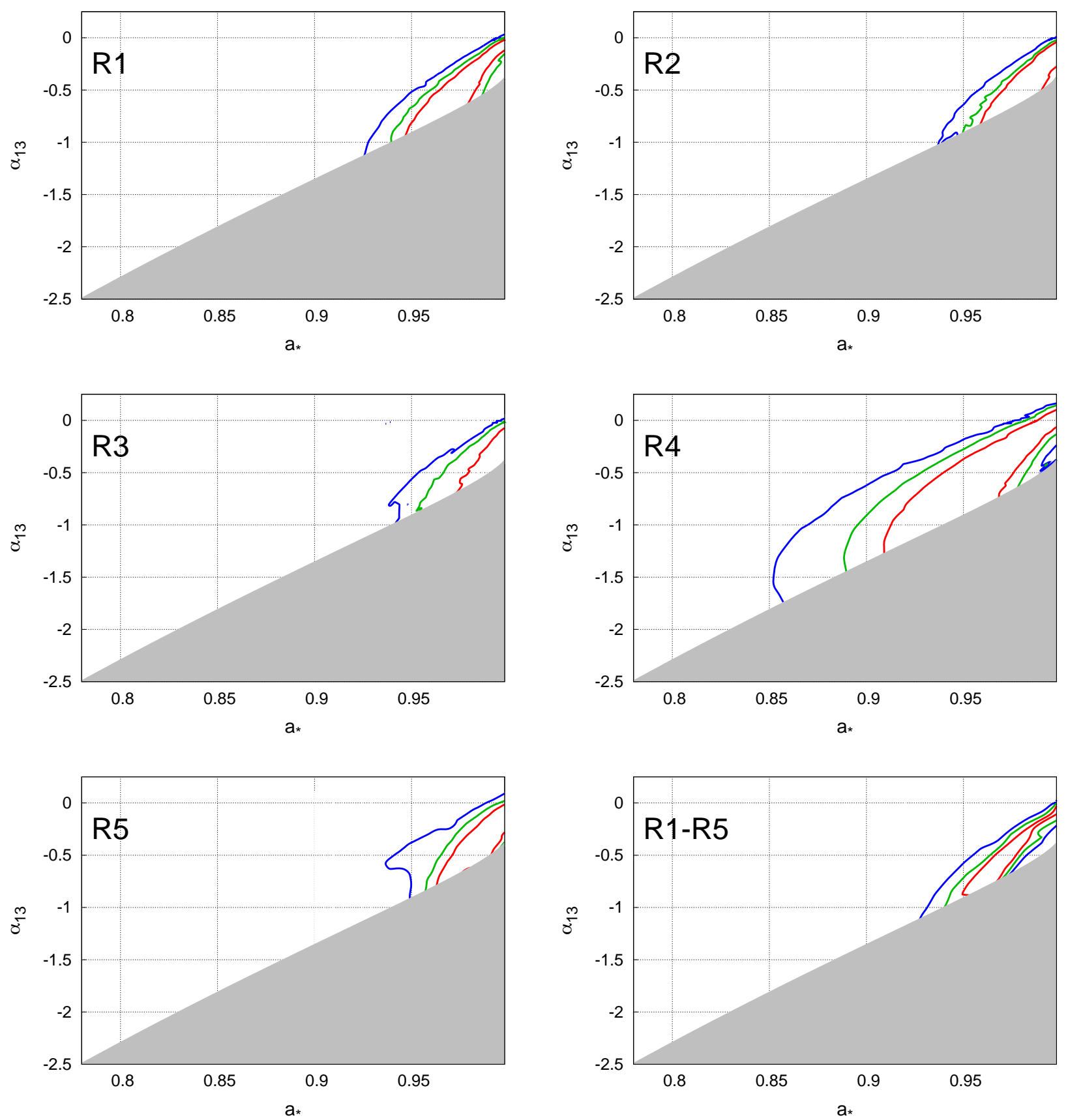

FIG. 4.- Constraints on the BH spin parameter $a_{*}$ and the deformation parameter $\alpha_{13}$ from the individual fits of the five RXTE observations of GRS 1915+105 (R1, R2, R3, R4, and R5) and from the fit of all observations together (R1-R5, bottom-right panel). The red, green, and blue curves represent, respectively, the $68 \%, 90 \%$, and $99 \%$ confidence level limits for two relevant parameters $\left(\Delta \chi^{2}=2.30\right.$, 4.61 , and 9.21, respectively). The gray region is ignored in our analysis because it violates the constraint in Eq. (A4) and the spacetime presents pathological properties there.

and we calculate the $\chi^{2}$ as

$$
\chi_{\text {tot }}^{2}=\chi_{\mathrm{RXTE}}^{2}+\chi_{\text {Suzaku }}^{2}+\frac{\left(M_{*}-M\right)^{2}}{\sigma_{M}^{2}}+\frac{\left(D_{*}-D\right)^{2}}{\sigma_{D}^{2}},
$$

where $\chi_{\mathrm{RXTE}}^{2}$ and $\chi_{\text {Suzaku }}^{2}$ are, respectively, the $\chi^{2}$ contribution from the RXTE and Suzaku data, $M$ and $D$ are free parameters in the model, $M_{*}=12.4 M_{\odot}$ $\sigma_{M}=2 M_{\odot}, D_{*}=8.6 \mathrm{kpc}$, and $\sigma_{D}=2.0 \mathrm{kpc}$ following the measurements of $M$ and $D$ in Reid et al. (2014). As in the analysis of the RXTE data, the last two terms on the right hand side of Eq. (9) are intended to incorporate the uncertainties on $M$ and $D$ in quite a simple way. The fit leads to the constraints on $a_{*}$ and $\alpha_{13}$ shown in Fig. 7. We recover Kerr and the constraints on $a_{*}$ and $\alpha_{13}$ are only slightly better than the constraints inferred from the sole analysis of the Suzaku spectrum. The estimate 


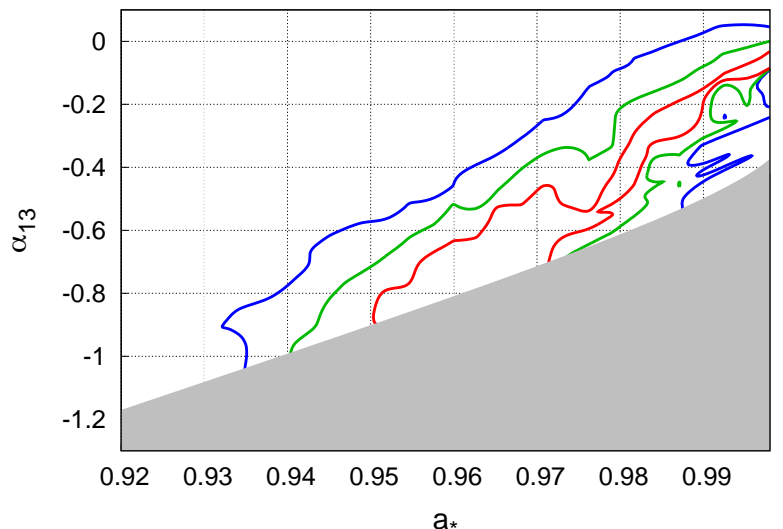

FIG. 5.- Constraints on the BH spin parameter $a_{*}$ and the deformation parameter $\alpha_{13}$ from the fit of the five RXTE observations together and taking the uncertainties on the $\mathrm{BH}$ mass, distance, and inclination angle of the disk into account by using the $\chi^{2}$ in Eq. (7). The red, green, and blue curves represent, respectively, the $68 \%, 90 \%$, and $99 \%$ confidence level limits for two relevant parameters $\left(\Delta \chi^{2}=2.30,4.61\right.$, and 9.21 , respectively). The gray region is ignored in our analysis because it violates the constraint in Eq. (A4) and the spacetime presents pathological properties there.

of $\alpha_{13}$ is ( $90 \%$ confidence level)

$$
\alpha_{13}=0.12_{-0.14}^{+0.02} \text {. }
$$

As we have already stressed, the analysis of the reflection features can indeed provide much stronger constraints than the continuum-fitting method. However, the combination of the two techniques can improve the constraints and, more importantly, it represents an important check for the systematics, which is often difficult to take into account.

Fig. 8 shows all the currently available constraints on the deformation parameter $\alpha_{13}$ from stellar-mass $\mathrm{BHs}$ with different techniques ${ }^{8}$ : the constraints are from the analysis of reflection features with relxill_nk (green), from gravitational wave data (Cárdenas-Avendaño et al. 2020) (red), with the continuum-fitting method with nkbb (magenta), and from the analysis of reflection features and disk's thermal spectrum by using relxill_nk and nkbb. The sole analysis of the disk's thermal component (the case of LMC X-1 in Fig. 8) cannot constrain $\alpha_{13}$ well because of the strong degeneracy between $a_{*}$ and $\alpha_{13}$. However, the combination of the analysis of reflection features and disk's thermal spectrum can improve the constraining power of X-ray reflection spectroscopy and from Fig. 8 we can see that the constraints in blue are the most stringent ones. We could imagine to further improve these constraints if we could test the spacetime metric around accreting BHs with the measurement of the frequencies of QPOs. As of now, this is not yet possible, because the exact mechanism responsible for QPOs is unknown. However, most QPO models involve the spacetime metric and it is thus likely that in the future, once the origin of these QPOs will be understood, we will also be able to combine the QPO data

8 The references of the original measurements can be found in Zhang et al. (2021).

to further strengthen the constraints on the deformation parameters.

Acknowledgments - This work was supported by the Innovation Program of the Shanghai Municipal Education Commission, Grant No. 2019-01-07-00-07E00035, the National Natural Science Foundation of China (NSFC), Grant No. 11973019, and Fudan University, Grant No. JIH1512604. D.A. is supported through the Teach@Tübingen Fellowship. A.T., C.B., H.L., and V.G. are members of the International Team 458 at the International Space Science Institute (ISSI), Bern, Switzerland, and acknowledge support from ISSI during the meetings in Bern.

\section{APPENDIX}

\section{JOHANNSEN METRIC}

For convenience of the reader, we report here the expression of the Johannsen metric. More details can be found in the original paper Johannsen (2013). In Boyer-Lindquist-like coordinates, the line element is

$$
\begin{aligned}
d s^{2}= & -\frac{\tilde{\Sigma}\left(\Delta-a^{2} A_{2}^{2} \sin ^{2} \theta\right)}{B^{2}} d t^{2}+\frac{\tilde{\Sigma}}{\Delta A_{5}} d r^{2}+\tilde{\Sigma} d \theta^{2}-\frac{2 a\left[\left(r^{2}+a^{2}\right) A_{1} A_{2}-\Delta\right] \tilde{\Sigma} \sin ^{2} \theta}{B^{2}} d t d \phi \\
& +\frac{\left[\left(r^{2}+a^{2}\right)^{2} A_{1}^{2}-a^{2} \Delta \sin ^{2} \theta\right] \tilde{\Sigma} \sin ^{2} \theta}{B^{2}} d \phi^{2}
\end{aligned}
$$

where $M$ is the BH mass, $a=J / M, J$ is the BH spin angular momentum, $\tilde{\Sigma}=\Sigma+f$, and

$$
\Sigma=r^{2}+a^{2} \cos ^{2} \theta, \quad \Delta=r^{2}-2 M r+a^{2}, \quad B=\left(r^{2}+a^{2}\right) A_{1}-a^{2} A_{2} \sin ^{2} \theta .
$$

The functions $f, A_{1}, A_{2}$, and $A_{5}$ are defined as

$$
f=\sum_{n=3}^{\infty} \epsilon_{n} \frac{M^{n}}{r^{n-2}}, \quad A_{1}=1+\sum_{n=3}^{\infty} \alpha_{1 n}\left(\frac{M}{r}\right)^{n}, \quad A_{2}=1+\sum_{n=2}^{\infty} \alpha_{2 n}\left(\frac{M}{r}\right)^{n}, \quad A_{5}=1+\sum_{n=2}^{\infty} \alpha_{5 n}\left(\frac{M}{r}\right)^{n},
$$

where $\left\{\epsilon_{n}\right\},\left\{\alpha_{1 n}\right\},\left\{\alpha_{2 n}\right\}$, and $\left\{\alpha_{5 n}\right\}$ are four infinite sets of deformation parameters without constraints from the Newtonian limit and Solar System experiments. In the present study, we only consider the deformation parameter 


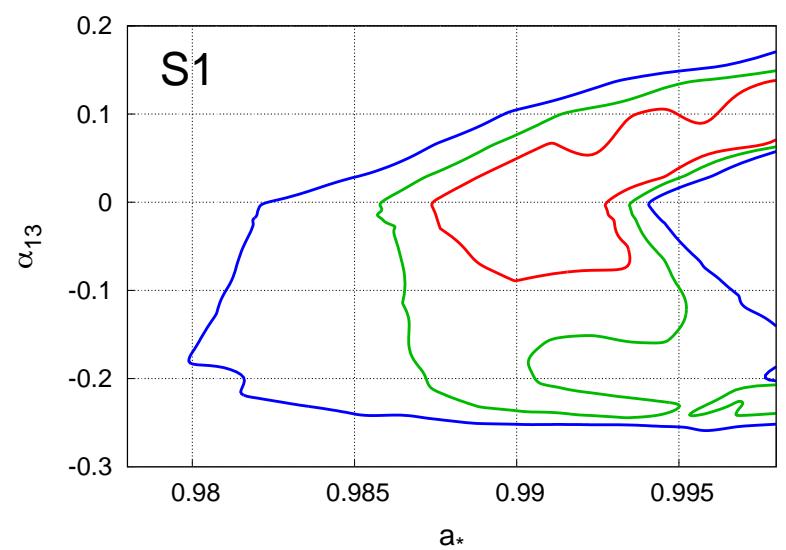

FIG. 6. - Constraints on the BH spin parameter $a_{*}$ and the deformation parameter $\alpha_{13}$ from the Suzaku observation of GRS $1915+105$. The red, green, and blue curves represent, respectively, the $68 \%, 90 \%$, and $99 \%$ confidence level limits for two relevant parameters $\left(\Delta \chi^{2}=2.30,4.61\right.$, and 9.21 , respectively).
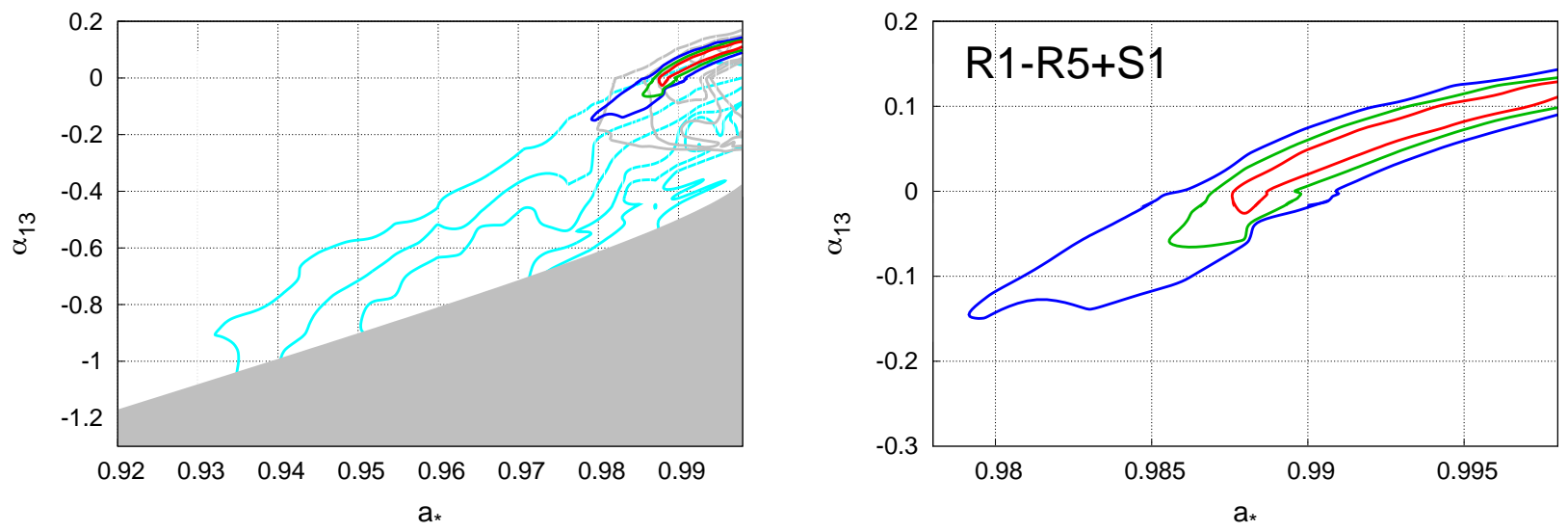

FIG. 7.- The left panel shows the constraints on the BH spin parameter $a_{*}$ and the deformation parameter $\alpha_{13}$ from the RXTE data (cyan curves and previously shown in Fig. 5), the Suzaku data (gray curves and previously shown in Fig. 6), and the combined analysis of the RXTE and Suzaku observations (where the red, green, and blue curves represent, respectively, the $68 \%, 90 \%$, and $99 \%$ confidence level limits for two relevant parameters). The right panel shows only the combined analysis of the RXTE and Suzaku observations.

$\alpha_{13}$, which has the strongest impact on the reflection spectrum, and we assume that all other deformation parameters vanish.

In order to avoid pathological properties in the spacetime, we have to impose some constraints on the values of $a_{*}$ and $\alpha_{13}$. For the $\mathrm{BH}$ spin, we require $\left|a_{*}\right| \leq 1$. This is the same constraint as in the Kerr spacetime: for $\left|a_{*}\right|>1$, there is no event horizon and the metric describes the spacetime of a naked singularity. For the deformation parameter $\alpha_{13}$, we require

$$
\alpha_{13}>-\frac{1}{2}\left(1+\sqrt{1-a_{*}^{2}}\right)^{4}
$$

\section{REFERENCES}

Abdikamalov, A. B., Ayzenberg, D., Bambi, C., et al. 2019, ApJ, 878, 91. doi:10.3847/1538-4357/ab1f89

Abdikamalov, A. B., Ayzenberg, D., Bambi, C., et al. 2020, ApJ, 899, 80. doi: $10.3847 / 1538-4357 /$ aba625

Alexander, S. \& Yunes, N. 2009, Phys. Rep., 480, 1. doi:10.1016/j.physrep.2009.07.002

Arnaud, K. A. 1996, Astronomical Data Analysis Software and Systems V, 101, 17

Bambi, C. 2011, Modern Physics Letters A, 26, 2453. doi:10.1142/S0217732311036929

Bambi, C. 2012, ApJ, 761, 174. doi:10.1088/0004-637X/761/2/174
Bambi, C. 2013, Phys. Rev. D, 87, 023007. doi:10.1103/PhysRevD.87.023007

Bambi, C. 2017, Reviews of Modern Physics, 89, 025001. doi:10.1103/RevModPhys.89.025001

Bambi, C. \& Barausse, E. 2011, ApJ, 731, 121. doi:10.1088/0004-637X/731/2/121

Bambi, C., Brenneman, L. W., Dauser, T., et al. 2020, arXiv:2011.04792

Bambi, C., Cárdenas-Avendaño, A., Dauser, T., et al. 2017, ApJ, 842, 76. doi:10.3847/1538-4357/aa74c0

Belloni, T., Klein-Wolt, M., Méndez, M., et al. 2000, A\&A, 355, 271 


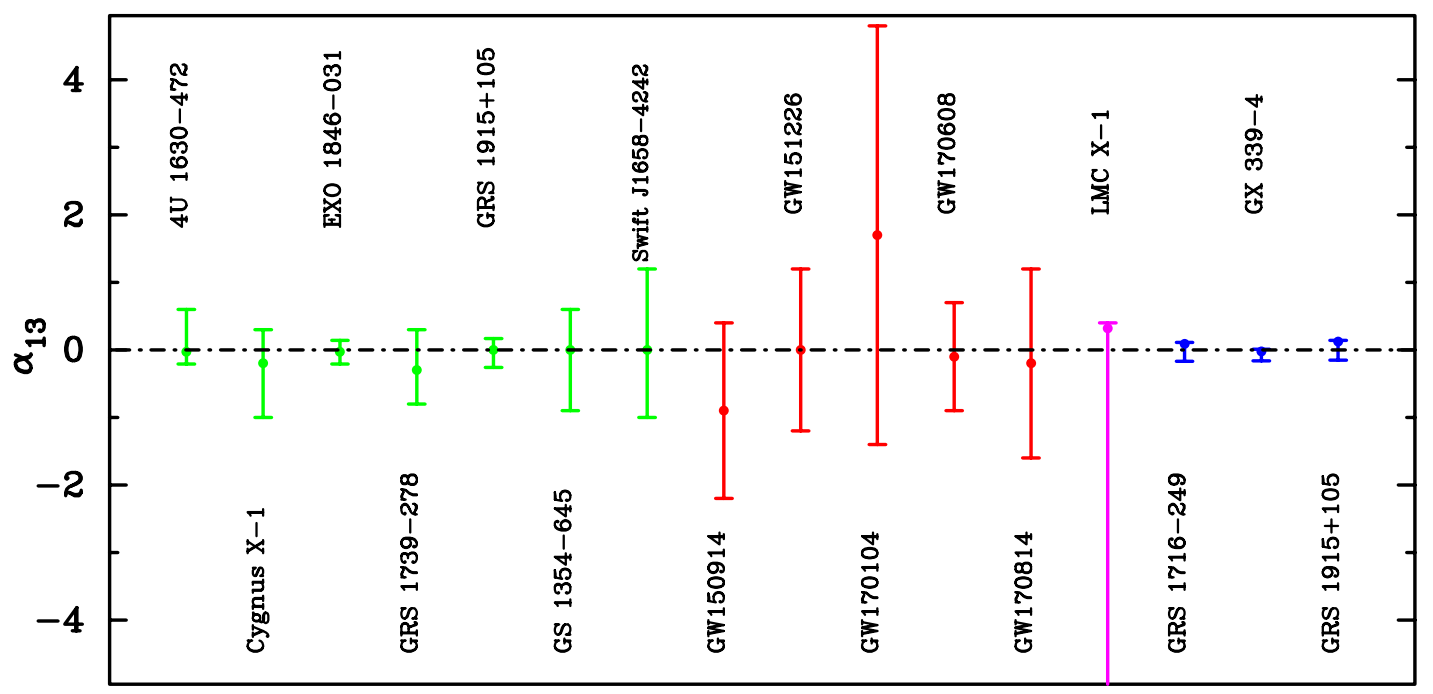

FIG. 8. - Summary of the available constraints on the Johannsen deformation parameter $\alpha_{13}$ from stellar-mass BHs. All error bars show the 3- $\sigma$ measurement. The constraints inferred from the analysis of reflection features are in green, those from gravitational wave data are in red, that from the continuum-fitting method in magenta, and the constraints obtained from the combination of the analysis of reflection features and the disk's thermal spectrum are in blue. See the text for more details.

Blum, J. L., Miller, J. M., Fabian, A. C., et al. 2009, ApJ, 706, 60. doi:10.1088/0004-637X/706/1/60

Brenneman, L. W. \& Reynolds, C. S. 2006, ApJ, 652, 1028. doi:10.1086/508146

Cárdenas-Avendaño, A., Nampalliwar, S., \& Yunes, N. 2020, Classical and Quantum Gravity, 37, 135008. doi:10.1088/1361-6382/ab8f64

Castro-Tirado, A. J., Brandt, S., \& Lund, N. 1992, IAU Circ., 5590

Chruściel, P. T., Costa, J. L., \& Heusler, M. 2012, Living Reviews in Relativity, 15, 7. doi:10.12942/lrr-2012-7

Cunningham, C. T. 1975, ApJ, 202, 788. doi:10.1086/154033

Dauser, T., Garcia, J., Wilms, J., et al. 2013, MNRAS, 430, 1694. doi:10.1093/mnras/sts710

Ebisawa, K., Ogawa, M., Aoki, T., et al. 1994, PASJ, 46, 375

Fender, R. P., Garrington, S. T., McKay, D. J., et al. 1999, MNRAS, 304, 865. doi:10.1046/j.1365-8711.1999.02364.x

García, J. \& Kallman, T. R. 2010, ApJ, 718, 695. doi:10.1088/0004-637X/718/2/695

García, J., Dauser, T., Lohfink, A., et al. 2014, ApJ, 782, 76. doi:10.1088/0004-637X/782/2/76

García, J. A., McClintock, J. E., Steiner, J. F., et al. 2014, ApJ, 794, 73. doi:10.1088/0004-637X/794/1/73

Giddings, S. B. 2017, Nature Astronomy, 1, 0067. doi:10.1038/s41550-017-0067

Herdeiro, C. A. R. \& Radu, E. 2014, Phys. Rev. Lett., 112, 221101. doi:10.1103/PhysRevLett.112.221101

Johannsen, T. 2013, Phys. Rev. D, 88, 044002. doi:10.1103/PhysRevD.88.044002

Johannsen, T. \& Psaltis, D. 2013, ApJ, 773, 57. doi:10.1088/0004-637X/773/1/57

Kleihaus, B., Kunz, J., \& Radu, E. 2011, Phys. Rev. Lett., 106, 151104. doi:10.1103/PhysRevLett.106.151104

Klein-Wolt, M., Fender, R. P., Pooley, G. G., et al. 2002, MNRAS, 331, 745. doi:10.1046/j.1365-8711.2002.05223.x

Kong, L., Li, Z., \& Bambi, C. 2014, ApJ, 797, 78. doi:10.1088/0004-637X/797/2/78

Kotani, T., Ebisawa, K., Dotani, T., et al. 2000, ApJ, 539, 413. doi:10.1086/309200

Lu, Y. \& Torres, D. F. 2003, International Journal of Modern Physics D, 12, 63. doi:10.1142/S0218271803002718

Martocchia, A., Matt, G., Karas, V., et al. 2002, A\&A, 387, 215. doi:10.1051/0004-6361:20020359
McClintock, J. E., Narayan, R., Davis, S. W., et al. 2011, Classical and Quantum Gravity, 28, 114009. doi:10.1088/0264-9381/28/11/114009

McClintock, J. E., Narayan, R., \& Steiner, J. F. 2014, Space Sci. Rev., 183, 295. doi:10.1007/s11214-013-0003-9

McClintock, J. E., Shafee, R., Narayan, R., et al. 2006, ApJ, 652, 518. doi:10.1086/508457

Miller, J. M., Parker, M. L., Fuerst, F., et al. 2013, ApJ, 775, L45. doi:10.1088/2041-8205/775/2/L45

Mirabel, I. F. \& Rodríguez, L. F. 1999, ARA\&A, 37, 409. doi:10.1146/annurev.astro.37.1.409

Mitsuda, K., Inoue, H., Koyama, K., et al. 1984, PASJ, 36, 741

Neilsen, J., Homan, J., Steiner, J. F., et al. 2020, ApJ, 902, 152. doi: $10.3847 / 1538-4357 /$ abb598

Parker, M. L., Tomsick, J. A., Kennea, J. A., et al. 2016, ApJ, 821, L6. doi:10.3847/2041-8205/821/1/L6

Psaltis, D., Perrodin, D., Dienes, K. R., et al. 2008, Phys. Rev. Lett., 100, 091101. doi:10.1103/PhysRevLett.100.091101

Reid, M. J., McClintock, J. E., Steiner, J. F., et al. 2014, ApJ, 796, 2. doi:10.1088/0004-637X/796/1/2

Remillard, R. A. \& McClintock, J. E. 2006, ARA\&A, 44, 49. doi:10.1146/annurev.astro.44.051905.092532

Reynolds, C. S. 2014, Space Sci. Rev., 183, 277. doi:10.1007/s11214-013-0006-6

Ross, R. R. \& Fabian, A. C. 2005, MNRAS, 358, 211. doi:10.1111/j.1365-2966.2005.08797.x

Schee, J. \& Stuchlík, Z. 2009, General Relativity and Gravitation, 41, 1795. doi:10.1007/s10714-008-0753-y

Shimura, T. \& Takahara, F. 1995, ApJ, 445, 780. doi:10.1086/175740

Titarchuk, L. 1994, ApJ, 434, 570. doi:10.1086/174760

Torres, D. F. 2002, Nuclear Physics B, 626, 377. doi:10.1016/S0550-3213(02)00038-X

Tripathi, A., Nampalliwar, S., Abdikamalov, A. B., et al. 2019, ApJ, 875, 56. doi:10.3847/1538-4357/ab0e7e

Tripathi, A., Liu, H., \& Bambi, C. 2020a, MNRAS, 498, 3565. doi:10.1093/mnras/staa2618

Tripathi, A., Zhou, M., Abdikamalov, A. B., et al. 2020b, ApJ, 897, 84. doi:10.3847/1538-4357/ab9600

Tripathi, A., Abdikamalov, A. B., Ayzenberg, D., et al. 2021a, ApJ, 907, 31. doi:10.3847/1538-4357/abccbd

Tripathi, A., Zhang, Y., Abdikamalov, A. B., et al. 2021b, ApJ, 913, 79. doi:10.3847/1538-4357/abf6cd 
Verner, D. A., Ferland, G. J., Korista, K. T., et al. 1996, ApJ, 465, 487. doi: $10.1086 / 177435$

Wilms, J., Allen, A., \& McCray, R. 2000, ApJ, 542, 914. doi:10.1086/317016

Zhang, S. N., Cui, W., \& Chen, W. 1997, ApJ, 482, L155. doi: $10.1086 / 310705$

Zhang, Y., Abdikamalov, A. B., Ayzenberg, D., et al. 2019a, ApJ, 875, 41. doi:10.3847/1538-4357/ab0e79

Zhang, Y., Abdikamalov, A. B., Ayzenberg, D., et al. 2019b, ApJ, 884, 147. doi:10.3847/1538-4357/ab4271
Zhang, Z., Liu, H., Abdikamalov, A. B., et al. 2021, arXiv:2106.03086

Zhou, M., Abdikamalov, A. B., Ayzenberg, D., et al. 2019, Phys. Rev. D, 99, 104031. doi:10.1103/PhysRevD.99.104031

Zhou, M., Abdikamalov, A. B., Ayzenberg, D., et al. 2020, MNRAS, 496, 497. doi:10.1093/mnras/staa1591 